\title{
Integrated On-demand Modeling for Configuration of Trusted ICT Supply Chains
}

\author{
Jānis Grabis $(\bowtie)$ \\ Department of Management Information Technology, Riga Technical University, \\ Kalku 1, Riga, Latvia \\ grabis@rtu. IV
}

\begin{abstract}
Digital enterprises and their networks increasingly rely on advanced decision-making capabilities, however, development of decision-making models requires significant effort and is often performed independently of other digitalization activities. Additionally, dynamic nature of many decision-making problems requires rapid ramp-up of decision-making capabilities. To addresses these challenges, this position paper proposes to elaborate a method for integrated ondemand decision modeling. The method combines mathematical programming and data analytics models to create case specific models on the basis of generic decision-making models. The integrated model and its data supply pipelines are configured using enterprise models allowing for consistent and rapid model deployment. The integrated model is intended for the trusted ICT supply chain configuration problem though it can be used for solving various types of decisionmaking problems. The main expected results are formulation of the new type decision-making model and the method for on-demand configuration of such models.
\end{abstract}

Keywords: On-demand modeling · Integrated modeling $\cdot$ ICT supply chain

\section{Introduction}

Digital enterprises increasingly rely on smart and intelligent decision-making based on non-trivial computations and complex algorithms (Carlsson 2018). Decision-making is perceived as a selection of business process execution alternatives (e.g., accept or decline a customer request in Customer Relationships Management system) or finding values of quantitative decision variables (i.e., how many products to order in Warehouse management System). It is assumed that decisions are made using a kind of algorithm or model referred as to decision-making model and the process of creating and using the model is referred as to decision-modelling. There are different types of decision-making problems, for example, capacity planning, supplier selection, fraud detection. Decision-making models have been developed for these typical decision-making problems, however these models still need to be adapted for specific use cases.

Decision-modelling often is time consuming and complex endeavor while dynamic operations increasingly require decision-making capabilities provided on short notice for solving short life-cycle decision-making problems (Halpern 2015), for example, 
selection of the delivery mode in hyperconnected systems (Sallez et al. 2016). That creates a need for on-demand decision-modelling (ODDM) allowing for quick model development, deployment and execution for a particular short-life cycle application case.

It is proposed that ODDM models can be developed by integrating various modelling paradigms. In particular, mathematical programming models are prescriptive models allowing to generate the optimal solution for the decision-making problem though requiring significant development expertise and effort. Data analytical models (e.g., regression, deep neural networks) on the other hand have predictive capabilities and given their structure and data can be estimated on-demand. Therefore, we propose to create ODDM models as integrated models or iODDM. On-demand models are envisioned to have multiple applications. The proposed research will focus specifically on configuration of Information and Communication Technology (ICT) supply chains (SC) what is a topical problem in practice (Kshetri and Voas 2019).

Challenges associated with intelligent applications and on-demand decision modelling can be summarized as follows:

1. Digital enterprises require rapidly deployable advanced decision-making models;

2. Models are still developed independently and their integration with other systems is done in an ad-hoc manner;

3. Data analytics models are more suitable for on-demand decision-making (e.g., non-parametric models) though some of data analytics models lack explanatory capabilities;

4. Model development is time consuming and performed on the case-to-case basis;

5. Many applications such as SC configuration are context dependent and require application case specific data structures.

To address these challenges, the goal of the proposed research is to enable development of on-demand decision-making models for configuration of trusted ICT SCs by integrating three modeling paradigms - enterprise modelling, mathematical programming and big data analysis. Enterprise models represent the decision-making problem in the enterprise context to facilitate integration, mathematical programming models capture underlying structure of the decision-modeling problem and big data analysis or data analytical models account for case specific variations in data structures and content. Expected scientific contributions are: 1) formulation of the general iODDM model and a new type of SC configuration model; 2) method for development of iODDM models as a part of decision-making information systems; 3) extension of enterprise modelling to support representation of trusted SC modelling aspects; and 4) big data processing pipeline for decision-modelling data supply.

\section{Related Work}

Integrated on-demand modeling and configuration of trusted ICT SC are two key areas of innovation of this proposal. Integrated and hybrid modelling is often used to attain benefits brought by different modelling paradigms (Chandra and Grabis 2016a). Complexity of current decision-making models also requires an appropriate development and execution environment (Chen and Zhang 2014). 
Models are typically integrated using a staged approach when one model serves as an input to another model (Chandra and Grabis 2016b). Optimization models are often combined with simulation models (Amaran et al. 2016). However, simultaneous running of mathematical programming and data analytical models is rarely considered. Kuo et al. (2015) provide one example where data analytical models feed mathematical programming models in real-time. The proposal's authors have made early attempts to integrate mathematical programming models and data analysis models (Grabis et al. 2012) though that is done without generalizing the integration approach. Ning and You (2019) point out that further development of integrated models requires better closed-loop feedback mechanisms. From the computational perspective, specification of decision-making components using domain specific languages allows development of reusable components pluggable in different enterprise applications (Brodsky et al. 2015). Wei and Ma (2014) integrate product configuration, production planning and production execution on the basis of the ERP system. Decision Model and Notation allows representing decisionmaking logics in business processes (Hasic et al. 2018). However, these technologies are often restricted to specific types of decision-models and do not cover the full-cycle of integrated modelling, especially, integration with enterprise modelling or on-demand modelling data provisioning. There is an increasing interest in modelling as a service though practical applications are mainly restricted to descriptive models (e.g., Schuff et al. 2018).

Mathematical and simulation models mainly have been used in SC configuration (Chandra and Grabis 2016b) and qualitative and descriptive techniques have been used to evaluate SC risks (Sigler et al. 2017). Patrignani and Kavathatzopoulos (2018) point out that complexity of ICT systems pose new challenges not addressed by the existing methods. Baryannis et al. (2019) conclude that artificial intelligence could help to address these concerns though comprehensive methods are yet to be developed.

The proposed research will extend the state of the art by elaborating a new type of integrated decision-making models, which fuse mathematical programming and data analytics in a generalized manner and are configurable according to enterprise models. The model when tailored to the trusted ICT SC configuration problem will be provide a novel solution to configuration of this type of SC using mathematical programming for typical SC configuration decisions and data analytics for risk and trustworthiness evaluation.

\section{Model Formulation}

A digital enterprise (or network of enterprises) face a decision-making problem (e.g., SC configuration). A generic decision-making model is available to solve this problem. The generic decision-making model needs to be tailored for a specific use case. The proposed method streamlines the tailoring process. Application of the method is supported by the decision support information system. Figure 1 shows the conceptual landscape of ODDM. The decision making problem is formally defined using enterprise modelling, which will be extended to cover needs specific to iODDM. The case specific model addressing the decision-making problem is developed as an iODDM consisting of the generic model and the data analytical model. It is configured according to the enterprise 
model to rise level of abstraction and improve integration with the decision support information system. The generic model captures core aspects of decision making and is formulated as a mathematical programming model while the data analytical model provides case specific customization using models fitted for the specific case.

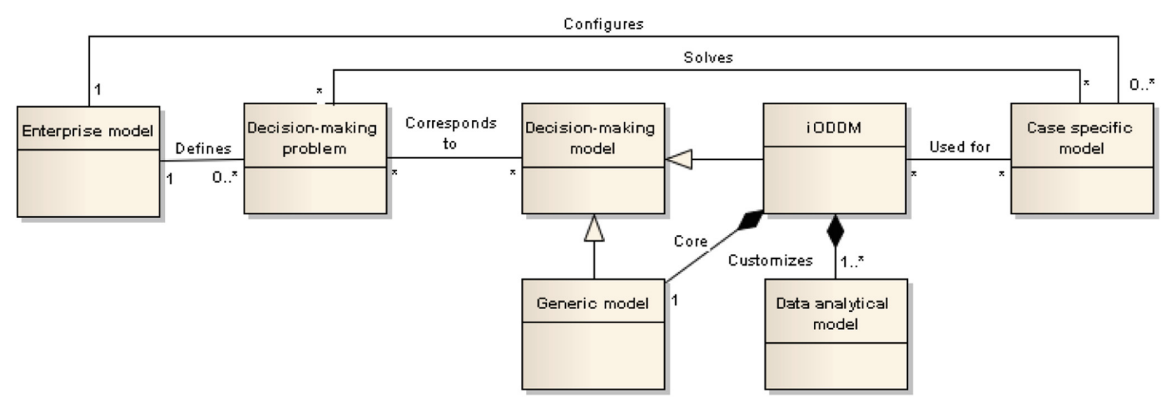

Fig. 1. Conceptual landscape of ODDM.

The integrated model combines enterprise modeling, mathematical programming and data analytics. Mathematical programming is used to represent the core part of the decision-making problem and data analytics is used to represent the case specific part of the decision-making problem. For instance, in the case of SC configuration, the mathematical programming model represents cost optimization and data analytical models are incorporated to represent influence of trust related aspects such supplier reputation, risk of delays and impact of shock events.

Figure 2 shows an initial proposal for the integrated SC configuration model. The SC configuration model deals with selection of suitable SC nodes, establishing connections among the nodes and allocating SC activities to the nodes and connections. In the case of ICT SCs, one needs to ensure that all components used in end-products and services meet security and reliability standards and requirements and SC can deliver products as promised withstanding various shock events. The core part of the model is a mathematical programming model. $\mathbf{X}, \mathbf{Y}$, and $\mathbf{V}$ are matrices representing decision variables concerning node selection, definition of connections and activity allocation, respectively. The vector $\mathbf{c}$ represents cost parameters associated with nodes, connections and activities as indicated by their indices (e.g., costs of operating a SC node). Vectors $\mathbf{a}_{\mathrm{i}}$ and $\mathbf{b}_{\mathrm{i}}$ are parameters used to specify constraints. The generic model is augmented by a customizable part. That includes customization of the objective function by adding a term $\mathbf{v}$ 'P, where $\mathbf{P}$ is a vector of penalties for not meeting case specific goals and $\mathbf{v}$ is a vector of weights indicating a relative importance of each goal. A corresponding set of constraints 4th item in Fig. 2 is also added to the model. These constraints represent relationships among target values of KPI and values estimated by the model. $\mathbf{k p i}^{\mathrm{T}}$ are target values set by decision-makers and $\mathbf{K P I}^{\mathrm{C}}$ is a KPI value estimated using the model. The penalty term in the objective function and the KPI constraint are added according to the enterprise model. Additionally, SC trustworthiness factor $\mathbf{R}$ is added to the objective and a constraint is added (3rd item in Fig. 2). The trustworthiness factor is a combination of security and trust factors characterizing the SC and it is evaluated using data 
analytical models. Various types of data analytical models could be used ranging from linear regression model to neural networks. The model can be estimated on-demand and represents security and reliability concerns relevant to particular case.

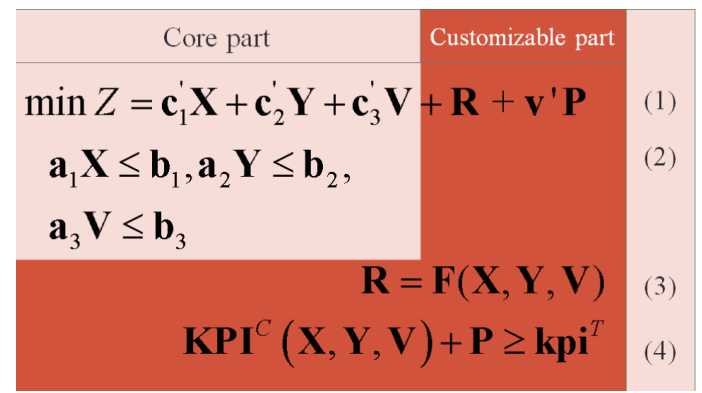

Fig. 2. The initial proposal of iODDM model.

The specific features to ICT SCs are representation of complex product structure, combination of virtual and physical aspects, and domain specific performance and quality attributes such as data security, performance and reliability attributes and licensing.

The enterprise model provides means for business analysts to configure iODDM. It represents the case specific goals and their KPI as well as restricts a number of plausible scenarios. For instance, configuration of SC for a new product has fewer constrains on decision variables than changing one of the nodes of the existing SC. The decisionmaking information system provides data needed for estimation and operation of data analytical models in a speedy and scalable manner.

It is important to note that data analytical models are an integral part of the model rather than just input data providers as it is a case in traditional multi-stage modeling. Results of the data analytical modeling are recalculated in every model solving iteration. Depending on the type of analytical models used traditional or non-parametric (e.g., genetic algorithms) will be used to solve the model.

\section{Modeling Process}

The iODDM model should be available for decision-making within a short time period measured from couple of minutes to one week. The method supports all steps of model development and includes three main stages: 1) Model development; 2) on-demand configuration; and 3) model execution and adaptation (Fig. 3). The model development stage concerns creation of the iODDM for the decision-making problem. Enterprise modeling techniques are used to specify modeling goals, constraints and data entities characteristic for the decision-making problem. The mathematical programming model is also formulated and it is linked with the enterprise model. The data entities represent data requirements for decision-making purposes. Usage of data analytical models is represented in an abstract form.

Whenever a need arises for an on-demand modeling, the model developed is configured in the second stage of the method. The enterprise model is changed to represent 


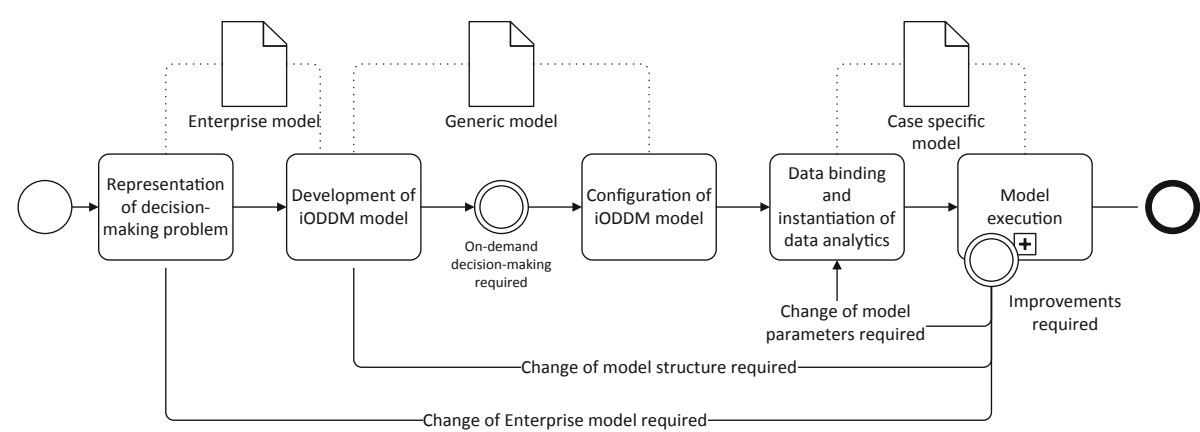

Fig. 3. Overview of the iODDM method.

case specific objectives and constraints and these changes are represented in the decisionmaking model by means of transformations. Case specific data sources are bound to the data requirements. That includes specification of data transformation procedures. It is important to note that both data streams and batch data can be used as data sources. The abstract data analytical models are instantiated using the case specific data and are made ready for usage in the optimization model.

The model execution stage concerns actual usage of the models. Two main challenges addressed during this stage are monitoring of data sources and scalability what is supported by the modeling platform. Additionally, adaptation of the modeling parameters also could be performed.

\section{Tool Support}

Development and execution of iODDM models is supported by the appropriate technological solution what is essential to deal with usability, scalability and reliability requirements of the iODDM method. The architecture of this decision-making information system or ODDM platform is given in Fig. 4.

It consists of the following key components:

- ODDM core - the central element of the architecture. It provides a web-based user interface that is used for iODDM model authoring and deployment. After models have been deployed and run various model related performance dashboards can be monitored in ODDM user interface. ODDM core also takes care of the infrastructure management and configuration of the remaining components of the ODDM architecture.

- Data ingest - used for ingesting data into the ODDM stream processor. Data ingestion in Fig. 4 is marked with \#1. Typical data sources are open data, internet of things and other types of data streams. The intelligent application itself can serve as a data source (e.g. passing in streams of log files or transactions).

- Stream processor - used for processing ingested data streams (\#2), which serve as the input for the iODDM model, and executing the model itself. Stream processor also aggregates the input data up to the defined level of granularity and persists it in the 


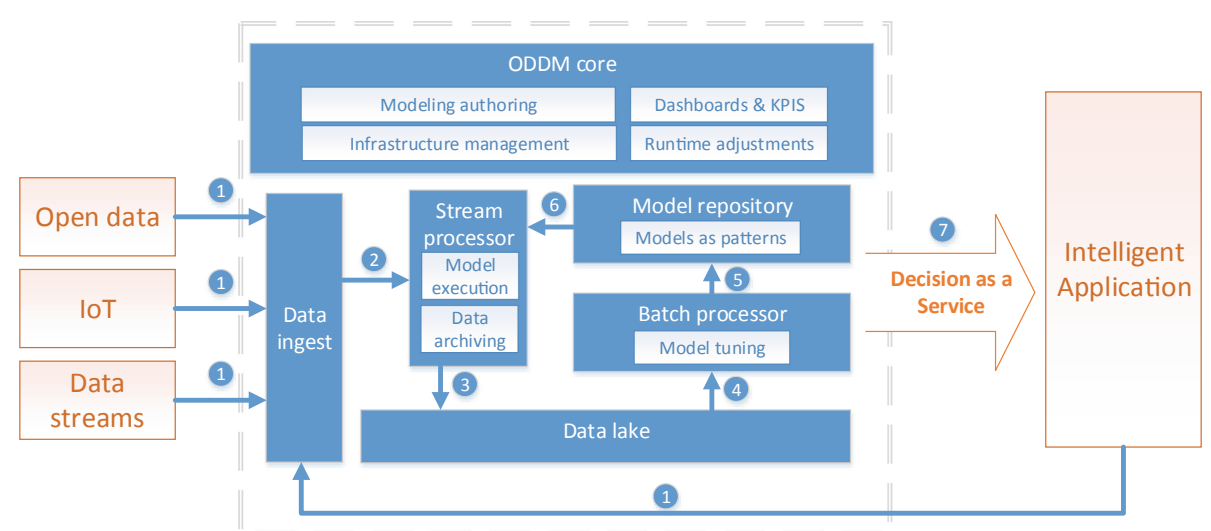

Fig. 4. Architecture of the ODDM platform.

data lake (\#3). During the model execution, Runtime adjustments are triggered to pass decision-modeling results to consumers, e.g., supply chain (\#7).

- Batch processor - provides batch processing of the archived data from the data lake (\#4) and fine tuning of model parameters which are then saved in the model repository (\#5). Various machine learning approaches are applied based on the available input data and model specifics.

- Model repository - provides storage of the integrated on-demand decision-making models in a form of reusable patterns that are then used by the Stream processor (\#6). The repository also accumulates models' usage performance data. Model performance is measured under specific contextual situation (defined by the input data) and Stream processor is able to switch to a more appropriate alternative model if the context would change.

- Data lake - a distributed, horizontally scalable persistent data store that integrates with the Stream processor (\#3) and batch data processor (\#4).

\section{Conclusion}

The paper describes an idea of creating iODDM models what includes both the modeling approach and tool support to enable complex computations required. The immediate steps of further research are development of case specific models and generalization of the observations made.

The proposed approach has a number of potential risks. That includes proving utility of using enterprise models to configure analytical models, data availability and stability of mathematical models with data modeling augmented constraints. Additionally, the proposed method relays having sufficient level of similarity among the case specific models and reusability of modeling components. 


\section{References}

Amaran, S., Sahinidis, N.V., Sharda, B., Bury, S.J.: Simulation optimization: a review of algorithms and applications). Ann. Oper. Res. 240(1), 351-380 (2016)

Brodsky, A., Krishnamoorthy, M., Menasce, D.A., Shao, G., Rachuri, S.: Toward smart manufacturing using decision analytics. In: Proceedings of 2014 IEEE International Conference on Big Data, p. 967 (2015)

Baryannis, G., Validi, S., Dani, S., Antoniou, G.: Supply chain risk management and artificial intelligence: state of the art and future research directions. Int. J. Prod. Res. 57(7), 2179-2202 (2019)

Carlsson, C.: Decision analytics-Key to digitalisation. Inf. Sci. 460, 424-438 (2018)

Chandra, C., Grabis, J.: Reconfigurable supply chains: an integrated framework. In: Chandra, C., Grabis, J. (eds.) SC Configuration, pp. 69-86. Springer, Heidelberg (2016a). https://doi.org/10. 1007/978-1-4939-3557-4_4

Chandra, C., Grabis, J.: Simulation modeling and hybrid approaches. In: Chandra, C., Grabis, J. (eds.) SC Configuration, pp. 173-197. Springer, Heidelberg (2016b). https://doi.org/10.1007/ 978-1-4939-3557-4_9

Chen, C.L.P., Zhang, C.-Y.: Data-intensive applications, challenges, techniques and technologies: a survey on big data. Inf. Sci. 275, 314-347 (2014)

Grabis, J., Chandra, C., Kampars, J.: Use of distributed data sources in facility location. Comput. Ind. Eng. 63(4), 855-863 (2012)

Halpern, F.: Next-generation analytics and platforms for business success: tDWI research report (2015). www.tdwi.org

Hasić, F., De Smedt, J., Vanthienen, J.: Augmenting processes with decision intelligence: principles for integrated modelling. Decis. Support Syst. 107, 1-12 (2018)

Kshetri, N., Voas, J.: Supply Chain Trust. IT Prof. 21(2), 6-10 (2019)

Kuo, Y., Leung, J.M.Y., Meng, H.M., Tsoi, K.K.F.: A real-time decision support tool for disaster response: a mathematical programming approach. In: Proceedings - 2015 IEEE International Congress on Big Data, BigData Congress 2015, p. 639 (2015)

Ning, C., You, F.: Optimization under uncertainty in the era of big data and deep learning: when machine learning meets mathematical programming. Comput. Chem. Eng. 125, 434-448 (2019)

Patrignani, N., Kavathatzopoulos, I.: On the complex relationship between ICT systems and the planet. In: Kreps, D., Ess, C., Leenen, L., Kimppa, K. (eds.) HCC13 2018, vol. 537, pp. 181-187. Springer, Heidelberg (2018). https://doi.org/10.1007/978-3-319-99605-9_13

Sallez, Y., Pan, S., Montreui, B., et al.: On the activeness of intelligent Physical Internet containers. Comput. Ind. 81, 96-104 (2016)

Schuff, D., Corral, K., St. Louis, R.D., Schymik, G.: Enabling self-service BI: a methodology and a case study for a model management warehouse. Inf. Syst. Front. 20(2), 275-288 (2018)

Sigler, K., Shoemaker, D., Kohnke, A.: Supply Chain Risk Management: Applying Secure Acquisition Principles to Ensure a Trusted Technology Product. CRC Press, Auburn Hills (2017)

Wei, J., Ma, Y.-S.: Design of a feature-based order acceptance and scheduling module in an ERP system. Comput. Ind. 65(1), 64-78 (2014) 